\title{
Anomalous features of the proximity effect in triplet superconductors
}

\author{
Y. Tanaka, ${ }^{1,2}$ Y. Asano, ${ }^{3}$ A. A. Golubov, ${ }^{4}$ and S. Kashiwaya ${ }^{5}$ \\ ${ }^{1}$ Department of Applied Physics, Nagoya University, Nagoya, 464-8603, Japan \\ ${ }^{2}$ CREST, Japan Science and Technology Corporation (JST) Nagoya, 464-8603, Japan \\ ${ }^{3}$ Department of Applied Physics, Hokkaido University, Sapporo 060-8628, Japan \\ ${ }^{4}$ Faculty of Science and Technology, University of Twente, 7500 AE, Enschede, The Netherlands \\ ${ }^{5}$ NeRI of National Institute of Advanced Industrial Science and Technology (AIST), Tsukuba, 305-8568, Japan
}

(Received 17 August 2005; published 21 October 2005)

\begin{abstract}
Anomalous features of the proximity effect specific to diffusive normal metal (DN) / triplet superconductor junctions are studied within the quasiclassical Green's function formalism. The pair amplitude $f_{N}$ as a function of energy $\varepsilon$ in DN, satisfies an anomalous relation, $f_{N}(\varepsilon)=-f_{N}^{*}(-\varepsilon)$, contrary to that in singlet superconductor junctions case where $f_{N}(\varepsilon)=f_{N}^{*}(-\varepsilon)$. Such an unusual $\varepsilon$ dependence is responsible for a zero energy peak in the local density of states and is a source of an anomalous penetration of the applied magnetic field into DN. Our results are relevant to $\mathrm{Sr}_{2} \mathrm{RuO}_{4}$, which is considered to have a $p_{x}+i p_{y}$-wave symmetry.
\end{abstract}

DOI: 10.1103/PhysRevB.72.140503

PACS number(s): 74.45.+c, 74.50.+r, 75.10.Pq

Nowadays spin-triplet superconductivity is a fascinating and an important issue in condensed matter physics. The existence of triplet superconductivity has become promising after a series of experiments in $\mathrm{Sr}_{2} \mathrm{RuO}_{4} \cdot{ }^{1,2}$ Other possible triplet superconductors are $\mathrm{UPt}_{3}$ (Ref. 3) and (TMTSF) ${ }_{2} \mathrm{X}^{4}$ Phase sensitive phenomena ${ }^{5}$ in junctions involving triplet superconductors is an important and relatively unexplored subject. One open question is how the proximity effect manifests itself in junctions between triplet superconductors and normal metals.

The proximity effect can be described in terms of the coherence between electrons and Andreev reflected holes in a normal metal. In unconventional superconductors, the internal phase reflecting sign change of a pair potential significantly affects charge transport since this sign change is a source of a midgap Andreev resonant state (MARS) at the surface of a superconductor. The origin of the MARS (Ref. 6) is the interference effect of a quasiparticle at the interface. ${ }^{7,8}$ In ballistic case, MARS leads to the formation of zero energy peak (ZEP) in local density of state (LDOS). However, the interference is destructive for $d$-wave superconductor /diffusive normal metal (DN) junctions ${ }^{9}$ and therefore no ZEP is expected in LDOS in DN. On the other hand, in diffusive normal metal / triplet superconductor (DN/TS) junctions the MARS penetrates into DN (Ref. 10) and ZEP occurs in the LDOS in DN.

Recently, a search of new phenomena in triplet superconductors has attracted a lot of attention. For example, recent experiments indicated possible existence of MARS in the tunneling spectroscopy of $\mathrm{Sr}_{2} \mathrm{RuO}_{4}$ and $\mathrm{Ru}$ microinclusion system. ${ }^{12}$ Therefore, it is important to explore the physics of proximity effect in DN/TS junctions and to address various situations relevant for the actual junctions.

In the present paper, we study the anomalous proximity effect in $N / \mathrm{DN} / \mathrm{TS}$ and TS/DN/TS junctions ( $N$ denotes normal electrode) in the framework of the Keldysh-Nambu quasiclassical Green's function formalism established in previous work. ${ }^{9-11}$ We find a crucial relation in pair amplitudes in $\mathrm{DN}, f_{N}(\varepsilon)=-f_{N}^{*}(-\varepsilon)$ in contrast to a relation $f_{N}(\varepsilon)=f_{N}^{*}(-\varepsilon)$, which holds in DN attached to a singlet superconductor. Here $\varepsilon$ is the quasiparticle energy measured from the Fermi level. The function $f_{N}(\varepsilon)$ is commonly used in the quasiclassical Green function theory and characterizes the pair amplitudes in DN due to the proximity effect. ${ }^{14}$ The property $f_{N}(\varepsilon)=-f_{N}^{*}(-\varepsilon)$ is responsible for the penetration of MARS into $N / \mathrm{DN} / \mathrm{TS}$ junctions which provides the ZEP in LDOS. Moreover, we predict an unusual response of DN to an external magnetic field. We show that the magnetic field spatially oscillates in DN instead of the usual Meissner screening. These unusual effects do not exist in the conventional proximity effect in singlet superconductors. We also show that in TS/DN/TS junctions, the proximity effect is suppressed when the external phase difference across the junction $\varphi$ is 0 due to the destructive interference of MARS's from the two superconductors. On the other hand, at $\varphi=\pi$ the constructive interference bridges the two MARS's in DN.

The model of the system is shown in Fig. 1. We consider a junction consisting of a normal metal and a superconducting reservoir connected by a quasi-one-dimensional diffusive conductor (DN) with a length $L$ much larger than the mean free path. The interface between DN and TS has a resistance $R_{b}$ while the DN/ $N$ interface has a resistance $R_{b^{\prime}}$. We assume flat interfaces and the insulating barriers at the two interfaces are modeled by the delta function. ${ }^{11}$ We start from the case where the pair potential is chosen to be $p_{x}+i p_{y}$-wave pairing symmetry, ${ }^{13}$ which is the promising candidate of superconductivity in $\mathrm{Sr}_{2} \mathrm{RuO}_{4} \cdot{ }^{1}$ As regards the spin structure of the

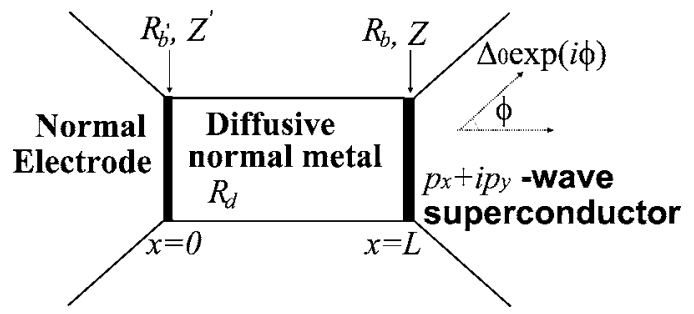

FIG. 1. Schematic illustration of the model of the N/DN/TS junction with $p_{x}+i p_{y}$-wave pairing 
Cooper pair, we choose $S_{z}=0$. The positions of the DN/N interface and the DN/TS interface are denoted as $x=0$ and $x=L$, respectively. We calculate the Keldysh-Nambu quasiclassical Green's functions by using the general boundary conditions given in Eqs. (4) and (5) in Ref. 11. To calculate the LDOS in DN, we focus on the retarded part of the Nambu-Keldysh Green's functions. For $p_{x}+i p_{y}$ wave pairing symmetry, the quasiclassical Green's function in TS is given by

$$
\hat{R}_{2 \pm}=f_{1 \pm} \hat{\tau}_{1}+f_{2 \pm} \hat{\tau}_{2}+g \hat{\tau}_{3}
$$

with $f_{1 \pm}=\Delta_{1, \pm} / \sqrt{\Delta_{0}^{2}-\varepsilon^{2}}, f_{2 \pm}=-\Delta_{2, \pm} / \sqrt{\Delta_{0}^{2}-\varepsilon^{2}}, g=\varepsilon / \sqrt{\varepsilon^{2}-\Delta_{0}^{2}}$, where $\Delta_{1 \pm}$ and $\Delta_{2 \pm}$ are given by $\Delta_{1 \pm}= \pm \Delta_{0} \cos \phi$ and $\Delta_{2 \pm}$ $=\Delta_{0} \sin \phi$, respectively. Here $\Delta_{1,+}+i \Delta_{2,+}$ and $\Delta_{1,-}+i \Delta_{2,-}$ are the effective pair potentials for a quasiparticle with an injection angle $\phi$ and $(\pi-\phi)$, respectively. The Green's function in DN is expressed by

$$
\hat{R}_{N}(x)=\sin \theta \hat{\tau}_{2}+\cos \theta \hat{\tau}_{3}
$$

with the proximity parameter $\theta$. Since the $\hat{\tau}_{3}$ component of the matrix current in Eq. (4) of Ref. 11 vanishes due to the absence of the Josephson current after the angular average of the matrix current as a function of $\varphi$, the $\hat{\tau}_{1}$ component of the Green's function in DN is vanishing. As discussed in Ref. 11, function $\theta$ in $\mathrm{DN}$ is determined by the Usadel equation

$$
D \frac{\partial^{2}}{\partial x^{2}} \theta+2 i \varepsilon \sin \theta=0
$$

supplemented by the boundary conditions ${ }^{9,15,16}$

$$
\left.\frac{L R_{b}}{R_{d}}\left(\frac{\partial \theta}{\partial x}\right)\right|_{x=L_{-}}=\left\langle F_{1}\right\rangle,\left.\quad \frac{L R_{b^{\prime}}}{R_{d}}\left(\frac{\partial \theta}{\partial x}\right)\right|_{x=0_{+}}=\left\langle F_{2}\right\rangle,
$$

where

$$
\begin{gathered}
F_{1}=\frac{4 T_{1}\left[\left(1+T_{1}^{2}\right)\left(A-B f_{1} i\right)+2 T_{1}\left(A B-i f_{1}\right)\right]}{\left[\left(1+T_{1}^{2}\right)+2 T_{1} B\right]^{2}-\left(1-T_{1}^{2}\right)^{2} f_{1}^{2}}, \\
F_{2}=\frac{2 T^{\prime} \sin \theta_{0}}{2-T^{\prime}+T^{\prime} \cos \theta_{0}},
\end{gathered}
$$

with $A=\left(\cos \theta_{L} f_{2}-\sin \theta_{L} g\right), \quad B=\left(\sin \theta_{L} f_{2}+\cos \theta_{L} g\right), \quad T_{1}$ $=T_{\phi} /\left(2-T_{\phi}+2 \sqrt{1-T_{\phi}}\right)$ and $T^{\prime}=T_{\phi}^{\prime}$.

Here $D$ is the diffusion constant in DN and $\langle\ldots\rangle$ denotes the average over angle $\phi$ defined as follows

$$
\left\langle F_{1(2)}(\phi)\right\rangle=\int_{-\pi / 2}^{\pi / 2} d \phi \cos \phi F_{1(2)}(\phi) / \int_{-\pi / 2}^{\pi / 2} d \phi T_{\phi} \cos \phi,
$$

$R_{d}$ is the resistance of DN, $\theta_{L}$ and $\theta_{0}$ are the values of $\theta$ at $x=L$ and $x=0$, respectively. In the above, $f_{1}=f_{1+}=-f_{1-}$ and $f_{2}=f_{2+}=f_{2-}$ are satisfied. The transparencies at the DN/TS and N/DN interface are given by

$$
T_{\phi}=\frac{4 \cos ^{2} \phi}{Z^{2}+4 \cos ^{2} \phi}, \quad T_{\phi}^{\prime}=\frac{4 \cos ^{2} \phi}{Z^{\prime 2}+4 \cos ^{2} \phi},
$$

where $Z$ and $Z^{\prime}$ denote the barrier parameter at the interfaces.
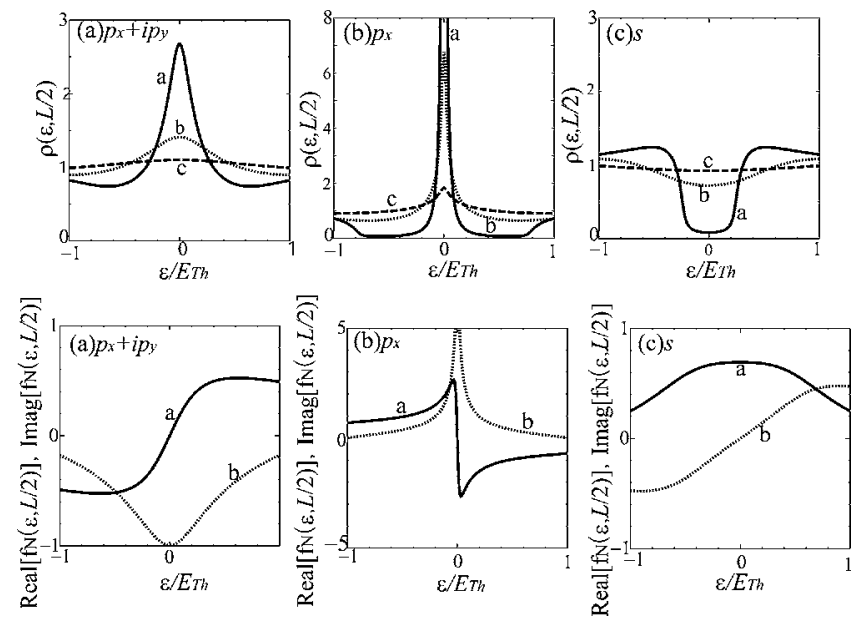

FIG. 2. In the upper panel, local density of states $\rho(\varepsilon, L / 2)$ is plotted for $N / \mathrm{DN} / \mathrm{S}$ junctions for various superconducting states with $Z=1, R_{d} / R_{b}=1, Z^{\prime}=1$, and $E_{T h}=0.25 \Delta_{0}$, respectively. (a) $p_{x}$ +ip $p_{y}$-wave, (b) $p_{x}$-wave, and (c) $s$-wave pairing. (a) $R_{d} / R_{b^{\prime}}=0.01$; (b) $R_{d} / R_{b^{\prime}}=1$; and (c) $R_{d} / R_{b^{\prime}}=100$. The corresponding pair amplitude $f_{N}(\varepsilon, L / 2)$ with $R_{d} / R_{b^{\prime}}=1$ are plotted for (a) $p_{x}+i p_{y}$-wave, (b) $p_{x}$-wave, and (c) $s$-wave pairing, respectively. (a) $\operatorname{Real}\left[f_{N}(\varepsilon, L / 2)\right]$, (b) $\operatorname{Imag}\left[f_{N}(\varepsilon, L / 2)\right]$.

At first we discuss the unusual $\varepsilon$ dependence of $\theta$. It is easy to confirm relations $f_{1(2)}(-\varepsilon, \phi)=f_{1(2)}^{*}(\varepsilon, \phi), g(-\varepsilon, \phi)$ $=g^{*}(\varepsilon, \phi), f_{1}(\varepsilon, \phi)=f_{1}(\varepsilon,-\phi), f_{2}(\varepsilon,-\phi)=-f_{2}(\varepsilon,-\phi)$, and $g(\varepsilon,-\phi)=g(\varepsilon, \phi)$ by the definition of $f_{1,2}$ and $g$. By applying these relations to Eqs. (4)-(6), we immediately find

$$
\theta(-\varepsilon)=-\theta^{*}(\varepsilon) .
$$

It can be shown from Eq. (22) in Ref. 11 that this equation holds for triplet junctions when time reversal symmetry is preserved. ${ }^{11}$ We note that $\theta(-\varepsilon)=\theta^{*}(\varepsilon)$ (Ref. 14) is satisfied in any singlet junction which preserves the time reversal symmetry. Thus, Eq. (9) is an important and a unique relation in triplet junctions. This relation leads to unusual properties of the proximity effect in triplet junctions. In what follows, we explicitly describe $x$ in $\rho(\varepsilon, x)$ and $f_{N}(\varepsilon, x)$ $=\sin \theta(\varepsilon, x)$. In Fig. 2, the local density of states $\rho(\varepsilon, x)$ $=\operatorname{Real}[\cos \theta(\varepsilon, x)]$ and $f_{N}(\varepsilon, x)$ with $\theta(\varepsilon, x)=\theta(\varepsilon)=\theta$ at the center of DN (i.e., $x=L / 2$ ) in N/DN/TS junctions are plotted for $p_{x}$ and $p_{x}+i p_{y}$, where $Z=1, Z^{\prime}=1$, and we fix a ratio $R_{d} / R_{b}$ at 1 . The interface resistance at $N / \mathrm{DN}\left(R_{b^{\prime}}\right)$ is a parameter of the calculation. In order to achieve the convergence of LDOS in numerical calculation, we replace $\varepsilon$ with $\varepsilon+i \gamma$ where $\gamma$ is small parameter, which we choose to be $0.005 \Delta_{0}$. As shown in Fig. 2(a), the LDOS has a ZEP, which indicates that MARS due to the proximity effect exists deep in the DN. The height of the ZEP of LDOS increases with the decrease of $R_{d} / R_{b^{\prime}}$. This is because the pair amplitude $f_{N}(\varepsilon, x)$ is more strongly confined within DN for larger $R_{b^{\prime}}$. In $p_{x}$-wave pairing symmetry, the same tendency is found in $\rho(\varepsilon, L / 2)$ as shown in Fig. 2(b). In $s$-wave junctions, in contrast to the triplet case, the amplitude of LDOS around $\varepsilon$ $=0$ decreases with the decrease of $R_{d} / R_{b^{\prime}}$ as shown in (c). For small $R_{d} / R_{b^{\prime}}$, quasiparticles in $N$ are separated from 


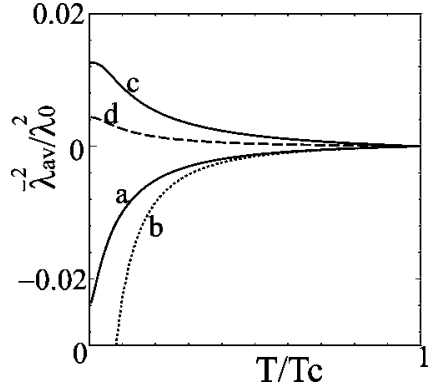

FIG. 3. Averaged value of the Meissner screening length $\bar{\lambda}_{a v}^{2}$ is plotted for various superconducting states with $Z=1, R_{d} / R_{b}=1$, $Z^{\prime}=1, R_{d} / R_{b^{\prime}}=1$, and $E_{T h}=0.25 \Delta_{0}$, respectively. (a) $p_{x}+i p_{y}$-wave, (b) $p_{x}$-wave, (c) $s$-wave, and (d) $d_{x^{2}-y^{2}}+i d_{x y}$-wave pairing.

those in DN and the minigap exists in DN. With the increase of $R_{d} / R_{b^{\prime}}$ the states within the minigap are filled in and the LDOS around $\varepsilon=0$ increases.

It is possible to understand the unusual $\varepsilon$ dependence of LDOS for $N / \mathrm{DN} / \mathrm{TS}$ junctions with $p_{x}$-wave symmetry in the limit $R_{d} / R_{b^{\prime}} \rightarrow 0$. We concentrate on the case with $0<\varepsilon \ll \Delta_{0}$ and $T_{\phi} \ll 1$. The spatial dependence of $\theta$ has a form $\theta=\theta_{L 0}+(x-L) \theta_{1} / L+(x-L)^{2} \theta_{2} /\left(2 L^{2}\right)$. Substituting $f_{1}$ $=\Delta_{0} \cos \phi / \sqrt{\Delta_{0}^{2} \cos ^{2} \phi-\bar{\varepsilon}^{2}}, f_{2}=0$, and $g=\bar{\varepsilon} / \sqrt{\bar{\varepsilon}^{2}-\Delta_{0}^{2} \cos ^{2} \phi}$ with $\bar{\varepsilon}=\varepsilon+i \gamma$ into Eq. (5), we find $\left\langle F_{1}\right\rangle \sim-\Delta_{0}\left(\sin \theta_{L 0}\right.$ $\left.+i \cos \theta_{L 0}\right) / \gamma$. Further, using Eqs. (3) and (4), we find $\cos \theta_{L 0}$ and $\rho(\varepsilon, x)$

$$
\cos \theta_{L 0} \sim \sqrt{\frac{\Delta_{0} R_{d} D}{4 i L^{2} R_{b} \gamma \varepsilon}}, \quad \rho(\varepsilon, x) \sim \sqrt{\frac{\Delta_{0} R_{d} D}{8 L^{2} R_{b} \gamma \varepsilon}} .
$$

It is remarkable that the $\rho(\varepsilon, x)$ is proportional to $\sqrt{1 /(\varepsilon \gamma)}$. This unusual $\varepsilon$ dependence is specific to DN/TS junctions.

In the lower panels of Fig. 2, the $\varepsilon$ dependence of the pair amplitude $f_{N}(\varepsilon, L / 2)$ is plotted for $R_{d} / R_{b}=1$. For triplet junctions in (a) and (b), the real and imaginary parts of $f_{N}(\varepsilon, L / 2)$ are odd and even functions of $\varepsilon$, respectively. On the other hand, in $s$-wave junctions, the real part of $f_{N}(\varepsilon, L / 2)$ is an even function of $\varepsilon$ and the imaginary part is an odd function of $\varepsilon$. These features are naturally understood from the fact that $\operatorname{Real}\left[f_{N}(\varepsilon, L / 2)\right]$ and $\operatorname{Imag}\left[f_{N}(\varepsilon, L / 2)\right]$ are, respectively, given by $\sin \theta_{r} \cosh \theta_{i}$ and $\cos \theta_{r} \sinh \theta_{i}$, where $\theta_{r}\left(\theta_{i}\right)$ is the real (imaginary) part of $\theta(\varepsilon, L / 2)$.

The unusual $\varepsilon$ dependence of $f_{N}(\varepsilon, x)$ in DN/TS junctions is a source of an anomalous response of DN to external magnetic field. When a magnetic field is applied parallel to the interface, the screening current $j(x)$ is given by ${ }^{17,18}$

$$
j(x)=\pi e^{2} N(0) D T \sum_{\omega_{n}} \operatorname{Tr}\left\{\hat{\tau}_{3} \hat{R}_{N}(x)\left[\hat{\tau}_{3}, \hat{R}_{N}(x)\right]\right\} A(x),
$$

where $A(x)$ is the vector potential, $N(0)$ is the density of states in the normal state at the Fermi level in DN, $\omega_{n}$ $=\pi T(2 n+1)$, and $T$ is the temperature. As a result, the magnetic field in the DN behaves as $H(x) \sim \exp [-x / \lambda(x)]$ with the local penetration depth $\lambda(x)$, which is given by
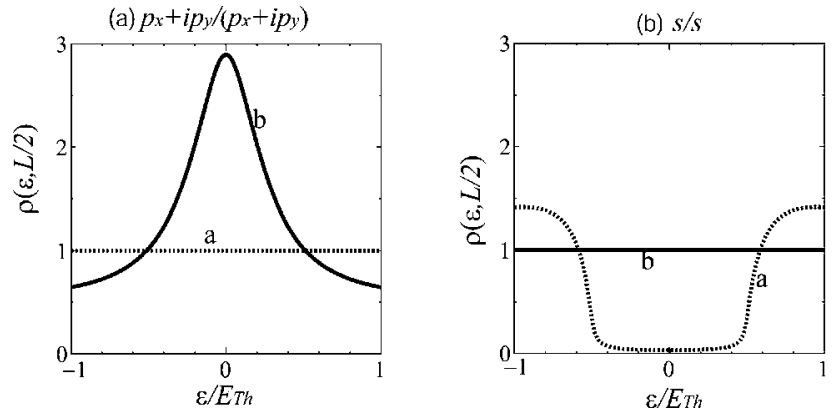

FIG. 4. Energy dependence of $\rho(\varepsilon, L / 2)$ is plotted for (a) $p_{x}$ $+i p_{y} / p_{x}+i p_{y}$ junctions and (b) $s / s$ junctions with $Z=Z^{\prime}=1, R_{d} / R_{b}$ $=R_{d} / R_{b^{\prime}}=1$, and $E_{T h}=0.25 \Delta_{0}$, respectively, for (a) $\varphi=0$ and (b) $\varphi$ $=\pi$.

$$
\frac{1}{\lambda^{2}(x)}=\frac{T \Sigma_{\omega_{n}} \sin ^{2} \theta\left(\omega_{n}\right)}{\lambda_{0}^{2}},
$$

as shown in Eq. (18) of Ref. 18 or Eq. (2.5) of Ref. 19 with $\lambda_{0}^{-2}=32 \pi^{2} e^{2} N(0) D T_{C}$, where $T_{C}$ is the transition temperature of TS. In Fig. 3, the averaged value of $\lambda^{2},\left[\bar{\lambda}_{a v}^{2}\right.$ $\left.=L / \int_{0}^{L} d x / \lambda^{2}(x)\right]$ is plotted as a function of temperatures $(T)$, where $Z=1, R_{d} / R_{b}=1, Z^{\prime}=1, R_{d} / R_{b^{\prime}}=1$, and $E_{T h}=0.25 \Delta_{0}$. As is shown in Fig. 3 by curves (c) and (d), $\bar{\lambda}_{a v}^{2}>0$ for singlet junctions. Thus $\bar{\lambda}_{a v}$ is a real number and a magnetic field is screened by the usual Meissner effect in the DN. On the other hand, in triplet junctions we find $\bar{\lambda}_{a v}^{2}<0$ as shown by curves (a) and (b). Therefore, the $\bar{\lambda}_{a v}$ becomes a purely imaginary number for $p_{x}+i p_{y^{-}}$and $p_{x}$-wave junctions. Formally, this is the consequence of the fact that the pair amplitude $f_{N}\left(i \omega_{N}, x\right)$ is purely imaginary. It is a feature of the anomalous proximity effect specific to triplet junctions that the applied magnetic field is not screened in the DN region and is screened only by the TS part.

Let us now discuss the proximity effect in TS/DN/TS junctions by replacing normal electrode (see Fig. 1) by TS. We study the effect of an external phase difference $\varphi$ of the $p_{x}+i p_{y}$-wave pair potentials on both sides of the junction on the LDOS in the DN layer. We assume that the barriers at $x=0$ and $x=-L$ are the symmetric (i.e., $R_{b^{\prime}}=R_{b}$ and $Z^{\prime}=Z$ ). We consider the two situations, $\varphi=0$ and $\varphi=\pi$. The boundary condition at $x=0$ is

$$
\left.\frac{L R_{b^{\prime}}}{R_{d}}\left(\frac{\partial \theta}{\partial x}\right)\right|_{x=0_{+}}=\left\langle F_{2}^{\prime}\right\rangle
$$

where $F_{2}^{\prime}$ is given by

$$
F_{2}^{\prime}=-\frac{4 T_{1}\left[\left(1+T_{1}^{2}\right)\left(A^{\prime}-B^{\prime} f_{1}^{\prime} i\right)+2 T_{1}\left(A^{\prime} B^{\prime}-i f_{1}^{\prime}\right)\right]}{\left[\left(1+T_{1}^{2}\right)+2 T_{1} B^{\prime}\right]^{2}-\left(1-T_{1}^{2}\right) f_{1}^{\prime 2}}
$$

with $A^{\prime}=\left(\cos \theta_{0} f_{2}^{\prime}-\sin \theta_{0} g\right)$ and $B^{\prime}=\left(\sin \theta_{0} f_{2}^{\prime}+\cos \theta_{0} g\right)$. For $\varphi=0$, we find $f_{1}^{\prime}=-f_{1}, f_{2}^{\prime}=f_{2}$ and for $\varphi=\pi, f_{1}^{\prime}=f_{1}, f_{2}^{\prime}=-f_{2}$, with $f_{1}=\Delta_{0} \cos \phi / \sqrt{\Delta_{0}^{2}-\varepsilon^{2}}$, and $f_{2}=\Delta_{0} \sin \phi / \sqrt{\Delta_{0}^{2}-\varepsilon^{2}}$. As in the case of $N / \mathrm{DN} / \mathrm{TS}$ junctions, to achieve the convergence 
of LDOS in numerical calculation, we replace $\varepsilon$ with $\varepsilon+i \gamma$ with small parameter $\gamma=0.005 \Delta_{0}$.

Here, we concentrate on the LDOS at the center of DN, $\rho(\varepsilon, L / 2)$, plotted in Fig. 4 for $\varphi=0$ and $\varphi=\pi$. As shown in Fig. 4(a), for $\varphi=0, \rho(\varepsilon, L / 2)=1$ in the whole $\varepsilon$ range. On the other hand, for $\varphi=\pi, \rho(\varepsilon, L / 2)$ has a clear ZEP. To understand this difference, we look at the property of $\theta$. By comparing the left and right boundary conditions using Eqs. (4) and (14), we can show for $\varphi=0$ that $\theta_{L}=-\theta_{0}$ and $d \theta /\left.d x\right|_{x=L_{-}}=d \theta /\left.d x\right|_{x=0_{+}}$. Then, the resulting pair amplitudes penetrating from the left side superconductor and the right side interfere destructively. Triplet pair potentials with $p_{x}$ $+i p_{y}$-wave symmetry change sign under spatial inversion. The phase difference of $\pi$ between the two pair amplitudes with MARS's penetrating from the two superconductors causes a destructive interference in DN in the absence of an external phase difference. On the other hand, for $\varphi=\pi$, the resulting $\theta$ satisfies $\theta_{L}=\theta_{0}$ and $d \theta /\left.d x\right|_{x=L_{-}}=-d \theta /\left.d x\right|_{x=0_{+}}$, and the interference becomes constructive in DN. The corresponding plot is also shown in the $s$-wave case. In this case, the constructive interference is enhanced for $\varphi=0$ and the resulting $\rho(\varepsilon, L / 2)$ has a minigap. On the other hand, for $\varphi$ $=\pi$, due to the destructive interference, the resulting $\rho(\varepsilon, L / 2)$ is unity due to the absence of the proximity effect at $x=L / 2$.
In this paper we have studied anomalous features of the proximity effect in triplet superconductor junctions in the framework of the quasiclassical Green's function formalism. We have shown that the pair amplitudes $f_{N}(\varepsilon)$ obey the unusual relation [i.e., $f_{N}(\varepsilon)=-f_{N}^{*}(-\varepsilon)$ in Eq. (9)] in contrast to that in singlet superconductor junctions. This unusual energy dependence induces the ZEP of LDOS in DN and is also responsible for the unusual behavior of a magnetic field in DN. The magnetic field is not screened in triplet junctions and oscillates spatially in DN. In N/DN/TS junctions, the magnitude of the ZEP increases with the increase of the interface resistance between $N$ and DN which is a result of the confinement of the MARS in the DN. Therefore, to observe the predicted ZEP in LDOS in experiments on $\mathrm{Sr}_{2} \mathrm{RuO}_{4}$ and $\mathrm{Ru}$ microinclusion systems a large resistance between $N$ and $\mathrm{DN}$ is necessary. In TS/DN/TS junctions, the proximity effect is suppressed when the external phase difference across junctions $\varphi=0$ because two MARS's penetrating from the two superconductors cancel each other. On the other hand, for $\varphi=\pi$, the constructive interference bridges the two MARS's in DN. These results imply the phase-sensitivity of MARS. Experimental observation of the predicted unusual proximity effect in triplet superconductor proximity systems would be of high interest.
${ }^{1}$ A. P. Mackenzie and Y. Maeno, Rev. Mod. Phys. 75, 657 (2003).

${ }^{2}$ K. D. Nelson, Z. Q. Mao, Y. Maeno, and Y. Liu, Science 306, 1151 (2004); Y. Asano, Y. Tanaka, M. Sigrist, and S. Kashiwaya, Phys. Rev. B 67, 184505 (2003); 71, 214501 (2005).

${ }^{3}$ H. Tou, Y. Kitaoka, K. Ishida, K. Asayama, N. Kimura, Y. Onuki, E. Yamamoto, Y. Haga, and K. Maezawa, Phys. Rev. Lett. 80, 3129 (1998).

${ }^{4}$ I. J. Lee, S. E. Brown, W. G. Clark, M. J. Strouse, M. J. Naughton, W. Kang, and P. M. Chaikin, Phys. Rev. Lett. 88, 017004 (2002).

${ }^{5}$ C. C. Tsuei and J. R. Kirtley, Rev. Mod. Phys. 72, 969 (2000); D. J. Van Harlingen, ibid. 67, 515 (1995).

${ }^{6}$ L. J. Buchholtz and G. Zwicknagl, Phys. Rev. B 23, 5788 (1981); J. Hara and K. Nagai, Prog. Theor. Phys. 74, 1237 (1986); C. Bruder, Phys. Rev. B 41, 4017 (1990); C. R. Hu, Phys. Rev. Lett. 72, 1526 (1994).

${ }^{7}$ Y. Tanaka and S. Kashiwaya, Phys. Rev. Lett. 74, 3451 (1995).

${ }^{8}$ S. Kashiwaya and Y. Tanaka, Rep. Prog. Phys. 63, 1641 (2000); T. Löfwander, V. S. Shumeiko, and G. Wendin, Supercond. Sci. Technol. 14, R53 (2001).

${ }^{9}$ Y. Tanaka, Y. V. Nazarov, and S. Kashiwaya, Phys. Rev. Lett. 90, 167003 (2003); Y. Tanaka, Y. V. Nazarov, A. A. Golubov, and S.
Kashiwaya, Phys. Rev. B 69, 144519 (2004).

${ }^{10}$ Y. Tanaka and S. Kashiwaya, Phys. Rev. B 70, 012507 (2004).

${ }^{11}$ Y. Tanaka, S. Kashiwaya, and T. Yokoyama, Phys. Rev. B 71, 094513 (2005).

${ }^{12}$ Z. Q. Mao, K. D. Nelson, R. Jin, Y. Liu, and Y. Maeno, Phys. Rev. Lett. 87, 037003 (2001); M. Kawamura, H. Yaguchi, N. Kikugawa, Y. Maeno, and H. Takayanagi, J. Phys. Soc. Jpn. 74, 531 (2005).

${ }^{13}$ M. Sigrist and K. Ueda, Rev. Mod. Phys. 63, 239 (1991).

${ }^{14}$ A. I. Larkin and Yu. V. Ovchinikov, Zh. Eksp. Teor. Fiz. 68, 1915 (1975) [Sov. Phys. JETP 41, 960 (1976); A. Scmidt and G. Schön, J. Low Temp. Phys. 20, 207 (1975).

${ }^{15}$ M. Yu. Kupriyanov and V. F. Lukichev, Zh. Eksp. Teor. Fiz. 94, 139 (1988) [Sov. Phys. JETP 67, 1163 (1988)].

${ }^{16}$ Yu. V. Nazarov, Superlattices Microstruct. 25, 1221 (1999); cond-mat/9811155.

${ }^{17}$ K. D. Usadel, Phys. Rev. Lett. 25, 507 (1970).

${ }^{18}$ W. Belzig, C. Bruder, and G. Schön, Phys. Rev. B 53, 5727 (1996).

${ }^{19}$ O. Narikiyo and H. Fukuyama, J. Phys. Soc. Jpn. 58, 4557 (1989). 